\title{
Outcome of living donor kidney transplant and deceased donor kidney transplant: a retrospective cohort study at national kidney and transplant institute
}

\author{
Abigail Burog, Adolfo Parayno
}

Department of Organ Transplantation and Vascular Surgery, National Kidney and Transplant Institute, Quezon City, Philippines

Background: A number of studies had been done in the Philippines on the outcome of deceased donor (DD) kidney transplantation but the studies comparing its outcome to the living donor (LD) kidney transplantation is still lacking. This study aims to determine the outcome of LD kidney transplantation and DD kidney transplantation in National Kidney and Transplant Institute.

Methods: This is a retrospective cohort study in a single tertiary training institution in the Philippines from January 2013 to December 2017. The graft function and graft and patient survival ratios at 6 months, 1 year, 2 years and 3 years were followed up. The DD and LD were compared using descriptive and logistic regression analysis to measure the association of the factors with graft and patient survival.

Results: There were 787 kidney donors, 154 DD and 633 LD. The mean age of donors were 31.1 years (9.5) while recipients were 42.3 years (12.2). There were 129 male DD (83.7\%) and 387 male LD (61.1\%). Three-year graft survival was 135 allografts (87.7\%) in DD and 599 allografts $(94.8 \%)$ in LD (odds ratio [OR], 0.39; 95\% confidence interval [Cl], 0.21-0.71; $\mathrm{P}<0.001)$. Three-year patient survival was 139 patients $(90.2 \%)$ in DD and 612 patients (96.8\%) in LD with $76 \%$ decrease of mortality $(\mathrm{OR}, 0.30 ; 95 \% \mathrm{Cl}, 0.15-0.61$; $\mathrm{P}<0.001)$.

Conclusions: There is a significant difference in patient and graft survival rates between the DD and LD. The 3-year graft survival and patient survival of DD kidney transplantation were lower compared to LD kidney transplantation. Despite the significant difference in the survival, the patient and graft survival were still high in both deceased and living donors and we still support the use of deceased donors for kidney transplant recipients.

Corresponding author: Abigail Burog

E-mail: abigail_burog@yahoo.com

(c) The Korean Society for Transplantation

This is an Open Access article distributed under the terms of the Creative Commons Attribution Non-Commercial License (http://creativecommons.org/licenses/by-nc/4.0/) which permits unrestricted non-commercial use, distribution, and reproduction in any medium, provided the original work is properly cited. 